\title{
A Preliminary Study of Milk Powder Hydration Using TEDSpiL Continuous Wave NMR
}

\author{
Steven T. Parslow ${ }^{1}$ | Najlaa K. Almazrouei ${ }^{1} \quad$ Michael I. \\ Newton $^{1 *}$ | Elizabeth R.Dye ${ }^{1}$ | Robert H. Morris ${ }^{1}$
}

${ }^{1}$ School of Science and Technology, Nottingham Trent University, Clifton Lane, Nottingham, NG11 8NS, UK

\section{Correspondence}

Michael I. Newton, School of Science and Technology, Nottingham Trent University, Clifton, Nottinghamshire, NG11 8NS, UK

Email: michael.newton@ntu.ac.uk

Funding information
Moisture content of foodstuffs are typically assessed by Titration or Near Infrared Spectroscopy which are labour intensive as a manual measurement or costly when automated. Magnetic Resonance offers a method for moisture evaluation but is also normally costly. In this work we revisit Look and Locker's "Tone Burst" experiment with a marginal oscillator to evaluate moisture content of powdered skimmed milk subjected to increased humidity. We refer to this technique as the Transient Effect Determination of Spin-Lattice (TEDSpiL) relaxation times. Moisture content in the samples ranged from 0-12\% as determined from the weight gained by the dry powder when re-suspended in water to reach a concentration of $40 \% \mathrm{w} / \mathrm{v}$. The relaxation properties of re-hydrated samples were measured with a CW NMR sensor. Solutions made up from powders with a higher retained moisture content provided lower measured relaxation values providing a method of measuring the moisture content of the powder. This technique provides a moisture measurement in under 5 seconds compared to several minutes for the equivalent pulsed method using low field hardware.

\section{KEYWORDS}

Continuous Wave, Spin-Lattice Relaxation, Milk Powder, Hydration, Moisture Content

*Corresponding Author 


\section{1 | INTRODUCTION}

The shelf life of milk can be extended by partial evaporation of the liquid fraction before spray drying into a vacuum vessel or rotodrying thus reducing the biological activity. The resulting powder is hygroscopic by nature and will readily absorb atmospheric moisture from its surroundings which will promote microbial growth and reduce the overall life and quality of the powder. The primary goal of storage is to limit the availability of atmospheric moisture. There are numerous methods for the measurement of water content in food [1, 2, 3, 4, 5, 6] such as Karl-Fischer Titration and Near Infrared Spectroscopy though these are challenging to automate, labour intensive or require significant cost investment in equipment. As water contains a large amount of hydrogen, Magnetic Resonance (MR) offers a useful tool for moisture measurement. Traditionally MR is often an expensive technique although increasingly low cost hardware is becoming available for such measurements.

Relaxation parameters in NMR are often measured using pulsed echo sequences such as inversion recovery for T1 and Carr-Purcel-Meiboom-Gill (CPMG) for T2. Inversion Recovery sequences can be time-intensive to operate although "one-shot" methods [7] are becoming more commonplace. With the dominance of pulsed NMR techniques, Continuous Wave (CW) Nuclear Magnetic Resonance (NMR) has been consigned mainly to student experiments and use in high sensitivity magnetometers. Whilst conceptually simpler, even with modern hardware, using CW NMR to determine relaxation parameters is not straight forward. However, the hardware for CW NMR can be an order of magnitude cheaper than a pulsed NMR system and requires significantly less power to operate. A standard CW NMR experiment involves measuring the RF power absorbed by a sample whilst it is swept through resonance either by varying the magnetic field (typically using so called sweep coils in addition to a static magnetic field as used here and shown in Figure 1) or varying the frequency of the RF source. So long as the rate of this sweep is short in comparison to $\mathrm{T}_{2}{ }^{*}$, the line-width of the resulting dip in RF power can be used to determine $\mathrm{T}_{2}{ }^{*}$. This is possible in highly homogeneous fields which are challenging to achieve whilst keeping the costs low and the system portable. For measurements of $\mathrm{T}_{1}$, Look and Locker [8] utilised a fixed frequency and gated sweep waveform they called a 'tone burst' where the sample was left aligned with the magnetic field but off resonance before the start of the measurement. Several cycles of the sweep coils follow during which each absorption trace was collected providing exponentially varying amplitudes with time constant $\mathrm{T}_{1}$ until saturation was reached. More recently [9] it was shown that a variation on this technique using a marginal oscillator could be used to produce a parameter related to $T_{1}$ which is termed $T_{x}$ and that calibration samples could then be used to relate this to $T_{1}$. We refer to this technique as the Transient Effect Determination of Spin-Lattice (TEDSpiL) relaxation times. The use of inexpensive NdFeB magnets and low-cost micro-controllers to perform this have also been reported [10] giving the potential of a relatively inexpensive, low power and portable system. This technique has the advantage of giving a value related to $T_{1}$ in under 5 seconds compared to several minutes for pulsed determination of $T_{1}$ with traditional low field hardware. In this work, we demonstrate that the parameter $T_{x}$ can be used to screen for moisture changes in re-hydrated milk from powders that have absorbed different levels of moisture during storage.

\section{2 | METHOD}

\section{1 | Equipment}

The continuous-wave NMR sensor used comprises two $0.5 \mathrm{~T} \mathrm{NbFeB}$ magnets mounted on an iron yoke (562 11 Leybold Didactic $\mathrm{GmbH}$, Germany) to generate a closed loop as shown in 1 . This arrangement provides a reasonably homogeneous field between the iron yokes corresponding to a larmor frequency for proton of 19.15Mhz. A commercial Radio Frequency (RF) coil is incorporated within an RF shield and forms a resonant circuit along with a marginal oscillator (P/N 


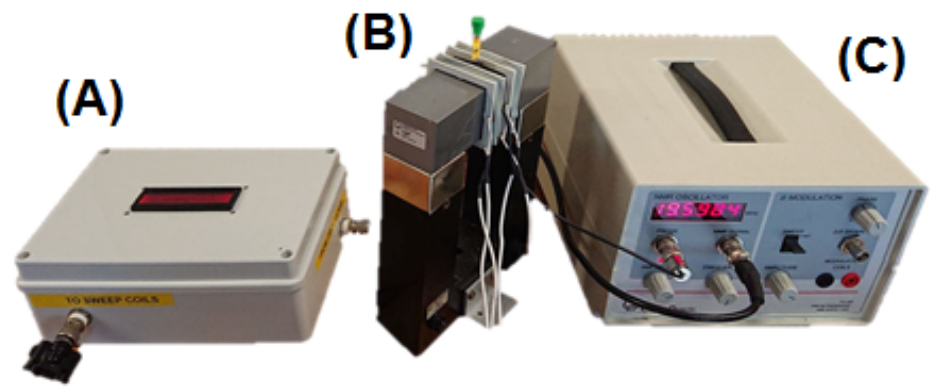

FIGURE 1 Cropped photograph of the experimental apparatus. (A) A plastic junction box housing the Teensy micro-controllers, MCP6002 inverting operational amplifier and batteries. (B) NMR sensor and sweep coils. (C) Leybold Marginal Oscillator.

514606 and 514602 respectively, both parts Leybold Didactic $\mathrm{GmbH}$, Germany). The setup also includes sweep coils to modulate the magnetic field. The RF power used to irradiate the sample is provided by the marginal oscillator unit and is fed into a micro-controller (Teensy 3.6, PJRC, OR, USA) which automatically fits the resulting peaks and displays the value of $T_{x}$ on a serial LCD screen (further explained in 2.3). A second micro-controller (Teensy 3.5, PJRC, OR, USA) was utilised to generate the ramp voltage which was amplified to drive the sweep coils. These sweep coils were set to $15 \mathrm{~V}$, providing an additional field between 0 and $13.5 \mathrm{mT}$. Using the $0.3 \mathrm{mT} / \mathrm{K}$ temperature drift reported[11], this sweep range compensates for fluctuations in field caused by thermal drift. A further in depth discussion of the measurement electronics has been reported [12].

\section{2 | Sample Preparation}

To measure the effect of milk powder concentration on measured $\mathrm{T}_{x}, 0.1 \%$ fat skimmed milk powder (Sainsbury's, UK) is mixed with distilled water to prepare test samples at six different concentrations $(5 \%, 10 \%, 15 \%, 20 \%, 25 \%$ and $30 \%$ $\mathrm{w} / \mathrm{v})$. These samples are loaded into NMR tubes before being placed into the $\mathrm{CW}$ sensor.

In order to measure the effect of moisture uptake by the milk powder on $T_{x}, 9$ additional $5 \mathrm{~g}$ samples of $0.1 \%$ fat skimmed milk powder are loaded into lid-less petri dishes and placed into a bench-top environmental chamber (3200, Thermotron, MI, USA) and subjected to various times under controlled humidity. The wetted powder is mixed with distilled water to a concentration of $40 \% \mathrm{w} / \mathrm{v}$ and moved to an ultrasound bath for 40 minutes to ensure it had been reasonably degassed after mixing. The water in the ultrasound bath is replaced with fresh room temperature water every 10 minutes to prevent heating the sample. These degassed samples are then loaded into NMR tubes before placing them into the $\mathrm{CW}$ sensor.

\section{3 | Measurement}

As the magnetic field is swept through resonance, an absorption event occurs characterised by a voltage change in the marginal oscillator (see example signal in Figure 2). These voltage values are passed into the analogue-to-digital converter ( $A D C$ ) in the Teensy micro-controller converting it to an ADC number. The maximum magnitude value from each absorption peak is stored in an array. The maximum magnitude for each sweep is processed with a least squares fit[13] after it has been converted to a natural logarithm value by the micro-controller. This process provides $a=m x+c$ 
fitting where the gradient is assigned as the value of $T_{x}$ for this specific sample. See Figure 2 for an example array of such data with the resulting fit.

Longitudinal relaxation times are measured using a selection of the hydrated milk powder solutions using a 1.5T clinical whole body MRI scanner (Magnetom Avanto, Siemens, Germany). These samples were imaged using a series of images collected with different inversion times. Similar voxels from each scan form an exponential decay which is fitted with in house Matlab (Mathworks, USA) routines to produce a $\mathrm{T}_{1}$ map.

\section{3 | PRELIMINARY EXPERIMENTS}

A $20 \mathrm{~g}$ sample of milk powder is stored in the environment chamber for 90 minutes to ensure a large uptake of moisture. Milk powder solutions are then made at varying concentrations (20\%, 25\%,30\%,35\% and $40 \% \mathrm{w} / \mathrm{v}$ ) with both the wet powder and a dry control powder. The $\mathrm{T}_{\mathrm{x}}$ of each concentration was compared for the wet and dry powder solutions to decide on the appropriate concentration to be used for future experiments. For concentrations under $40 \% \mathrm{w} / \mathrm{v}$, the difference in $T_{x}$ values between the wet and dry samples is marginal, but at $40 \% \mathrm{w} / \mathrm{v}$ the difference between wet and dry becomes significant. For this reason, $40 \% \mathrm{w} / \mathrm{v}$ solutions were used as the standard concentration for all future experiments.The data for this experiment is not shown.

A similar experiment is undertaken to find the most appropriate humidity value to which the powder should be exposed to give a good variation in moisture content in a reasonable time frame. A higher relative humidity corresponds to a faster uptake in atmospheric moisture as well as increasing the maximum amount of retained moisture. Samples are placed inside at $75 \%, 85 \%$ and $95 \%$ relative humidity, all at $20^{\circ} \mathrm{C}$. It was found that $85 \%$ relative humidity presents a good balance between maximum retained moisture and the rate of uptake and is used in future experiments. The data for this experiment is not shown.

Whilst the samples are being mixed with water, they become aerated which causes an unknown and variable change in the spin lattice relaxation time and hence $\mathrm{T}_{\mathrm{x}}$. It was found that placing the sample in an ultrasonic bath helped remove trapped air in the milk mixture assisting the reliability of measurements. A single cycle of 10 minutes in a $70 \mathrm{~W}$ ultrasonic jewellery cleaner bath removes a significant proportion of the additional gas but it was found that after four such cycles (40 minutes total in ultrasonic bath) resulted in a plateau of signal and standard deviation. As such, all samples are sonicated for 40 minutes.

\section{4 | RESULTS}

The variation in $T_{x}$ of solutions due to changes in the concentration of milk powder is demonstrated in Figure 3. Higher concentrations of milk powder in distilled water leads to an increase in viscosity due to the abundance of lactose, proteins and fats which is known to decrease spin-lattice relaxation times [14, 15], reproducing the results of [16].

When powders which have been subjected to longer duration in a humid environment are made up into a $40 \% \mathrm{w} / \mathrm{v}$ concentration solution, the measured $T_{X}$ decreases as can be seen in Figure 4. It is likely that this is simply due to less milk powder making up the $2 \mathrm{~g}$ weight, with some of it being water. As such, the true concentration will be lower than that which is measured. Through this concept it is possible to create a calibration set of known moisture levels in milk powders and use it to measure the amount of water in an unknown sample (prepared with weight to volume) to ensure it has been successfully and appropriately dehydrated. Furthermore, this measurement would be suitable for quality assurance of products comprising powdered milk solutions.

The $T_{1}$ map described in the previous section is shown in Figure 5 . It is found that the differences in $T_{1}$ is less than 
$50 \mathrm{~ms}$ and is outside the range which is statistically differentiated by such a measurement suggesting an advantage in terms of the sensitivity of the CW NMR method in comparison to traditional pulsed imaging techniques.

\section{5 | CONCLUSION}

CW-NMR has been demonstrated to distinguish between the moisture levels of skimmed milk powder when it is re-hydrated to a $40 \%$ solution by weight. The sensor and electronics used in this investigation are orders of magnitude cheaper than the industry standard of using an automated Karl Fischer titration system to measure the moisture level of the milk powder, proving a cost-effective analytical method that is easy and intuitive to use. All pieces of equipment and reagents were selected due to how readily purchasable they are requiring minimal modifications to rebuild a working system. A major advantage in using CW NMR with permanent magnets is that as the larmor frequency is swept, frequency changes that could arise from the thermal drift of the NeFeB magnets can be compensated for and therefore the device doesn't need to undergo re-tuning or thermal control measures. Any extreme changes in temperature can be compensated for easily by changing the fundamental frequency of the marginal oscillator tank circuit which is as simple as adjusting a variable capacitor. The sensitivity of CW NMR has also been compared to mainstream pulsed imaging techniques showing that it can more easily distinguish between smaller changes in $\mathrm{T}_{1}$ in this sample. This technique offers promise for online process monitoring for quality control purposes or as a standalone sampling system.

\section{ACKNOWLEDGEMENTS}

STP gratefully acknowledges Nottingham Trent University for funding under the Vice Chancellors Bursary Award.

\section{CONFLICT OF INTEREST}

There are no conflicts of interest for this work.

\section{REFERENCES}

[1] Reh C, Bhat SN, Berrut S. Determination of water content in powdered milk. Food Chemistry 2004;86(3):457 - 464. http://www.sciencedirect.com/science/article/pii/s0308814603005946.

[2] Isengard HD. Water determination : Scientific and economic dimensions. Food Chemistry 2008;106(4):1393 - 1398. http://www.sciencedirect.com/science/article/pii/s0308814607005845, 4th International Workshop on Water in Foods.

[3] Caro CAD, Aichert A, Walter CM. Efficient, precise and fast water determination by the Karl Fischer titration. Food Control 2001;12(7):431 - 436. http://www. sciencedirect.com/science/article/pii/s0956713501000202, water Determination in Food - a Challenge for the Analysts.

[4] Isengard HD. Water content, one of the most important properties of food. Food Control 2001;12(7):395 - 400. http: // www.sciencedirect.com/science/article/pii/s0956713501000433, water Determination in Food - a Challenge for the Analysts.

[5] Christie J, Platt IG. Moisture content: What is it and how can it be measured? In: 2014 IEEE Sensors Applications Symposium (SAS); 2014. p. 161-165.

[6] Rückold S, Grobecker KH, Isengard HD. Determination of the contents of water and moisture in milk powder. Fresenius' Journal of Analytical Chemistry 2000 oct;368(5):522-527. http: //link. springer . com/10. 1007/s002160000511. 
[7] Moraes TB, Monaretto T, Colnago LA. Rapid and simple determination of T1 relaxation times in time-domain NMR by CWFP sequence. Journal of Magnetic Resonance 2016;270:1-6. https://www. sciencedirect. com/science/article/ pii/s1090780716300957.

[8] Look DC, Locker DR. Nuclear Spin-Lattice Relaxation Measurements by Tone-Burst Modulation. Phys Rev Lett 1968 Apr;20:987-989. https://link.aps .org/doi/10.1103/PhysRevLett.20.987.

[9] Morris RH, Mostafa N, Parslow S, Newton MI. Transient effect determination of spin-lattice (TEDSpiL) relaxation times using continuous wave NMR. Magnetic Resonance in Chemistry 2017;55(9):853-855. https : //onlinelibrary . wiley . com/doi/abs/10.1002/mrc. 4594.

[10] Newton MI, Breeds EA, Morris RH. Advances in Electronics Prompt a Fresh Look at Continuous Wave (CW) Nuclear Magnetic Resonance (NMR). Electronics 2017;6(4). http: //www . mdpi . com/2079-9292/6/4/89.

[11] Hughes-Riley T, Dye ER, Anderez DO, Hill-Casey F, Newton MI, Morris RH. Temperature dependence of magnetic resonance probes for use as embedded sensors in constructed wetlands. Sensors and Actuators A: Physical 2016;241:19-26. https://www.sciencedirect.com/science/article/pii/s0924424716300504.

[12] Parslow ST, Newton MI, Morris RH. A microcontroller system for the automation of transient effect determination of the spin-lattice relaxation time using continuous wave NMR. Proceedings 2018;https://sciforum. net/manuscripts/ 5636/manuscript.pdf.

[13] Press WH, Teukolsky SA, Vetterling WT, Flannery BP. Numerical Recipes in C (2Nd Ed.): The Art of Scientific Computing. New York, NY, USA: Cambridge University Press; 1992.

[14] Todt H, Guthausen G, Burk W, Schmalbein D, Kamlowski A. Water/moisture and fat analysis by time-domain NMR. Food Chemistry 2006;96(3):436-440.

[15] Colsenet R, Mariette F, Cambert M. NMR Relaxation and Water Self-Diffusion Studies in Whey Protein Solutions and Gels. Journal of Agricultural and Food Chemistry 2005;53(17):6784-6790. https://doi .org/10.1021/jf050162k, pMID: 16104800.

[16] Almazrouei NK, Newton MI, Dye ER, Morris RH. Novel Food-Safe Spin-Lattice Relaxation Time Calibration Samples for Use in Magnetic Resonance Sensor Development. Proceedings 2018;2(3). http://www . mdpi . com/2504-3900/2/3/122.

\section{GRAPHICAL ABSTRACT}

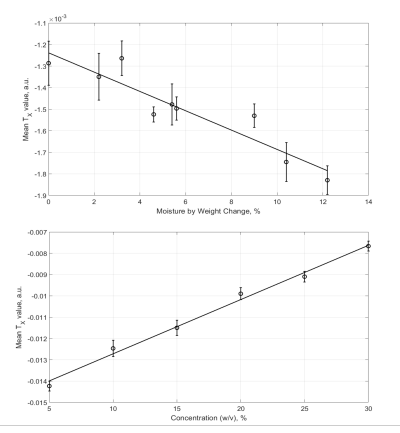

Continuous Wave Magnetic Resonance is suitable for sensitive detection of moisture content in reconstituted milk powder solutions prepared by weight. A fully automated system outputs a value which is proportional to the powder's moisture content before rehydration. This shows promise as a tool for online quality control or testing. 

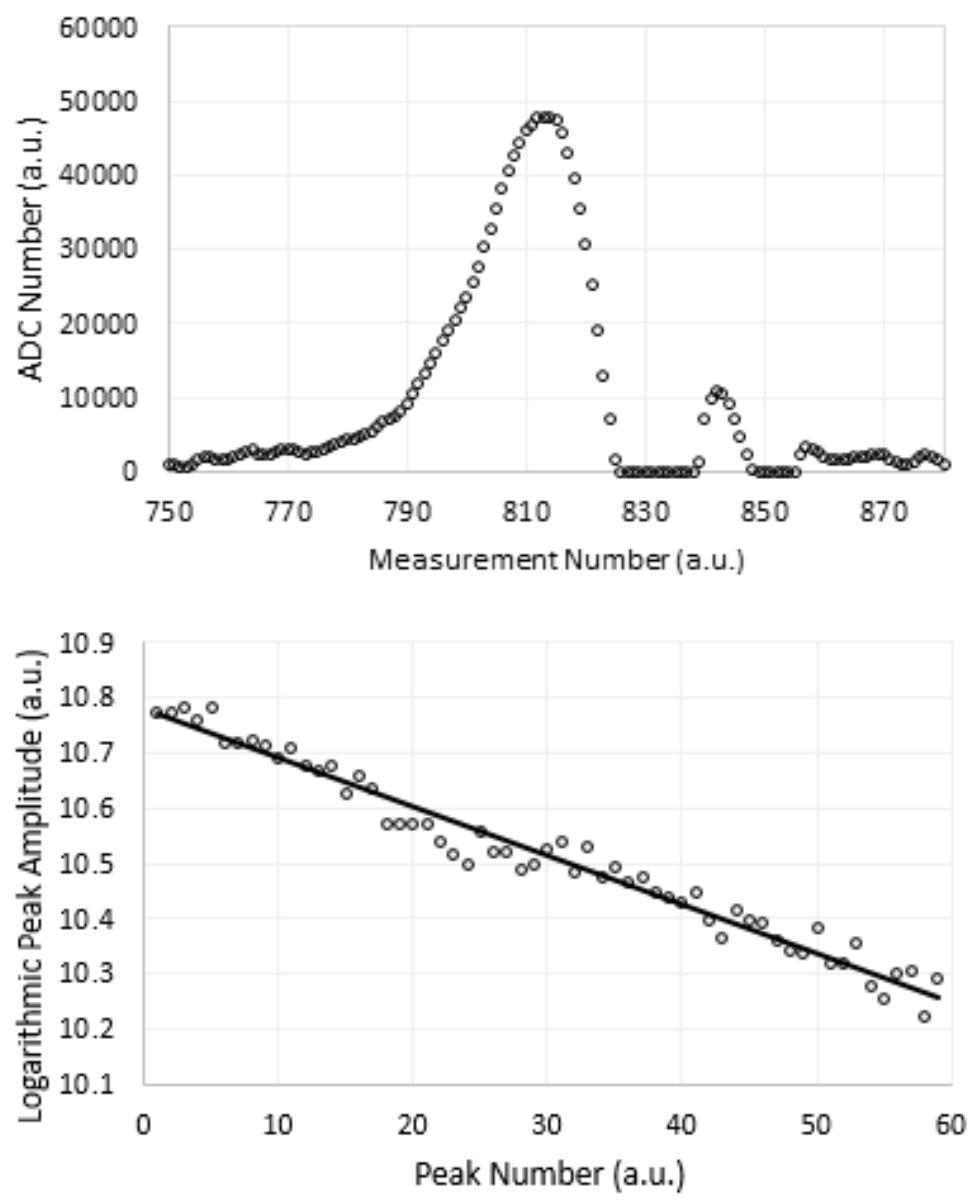

F I GURE 2 Top The Analogue to Digital Converter (ADC) values returned from the Teensy 3.6 for a single linear sweep through resonance. Bottom An example of the logarithmic peak values of water returned from the peak value array with a least squares fit. The gradient of the linear fit provides the numerical value to the parameter $T_{x}$. 


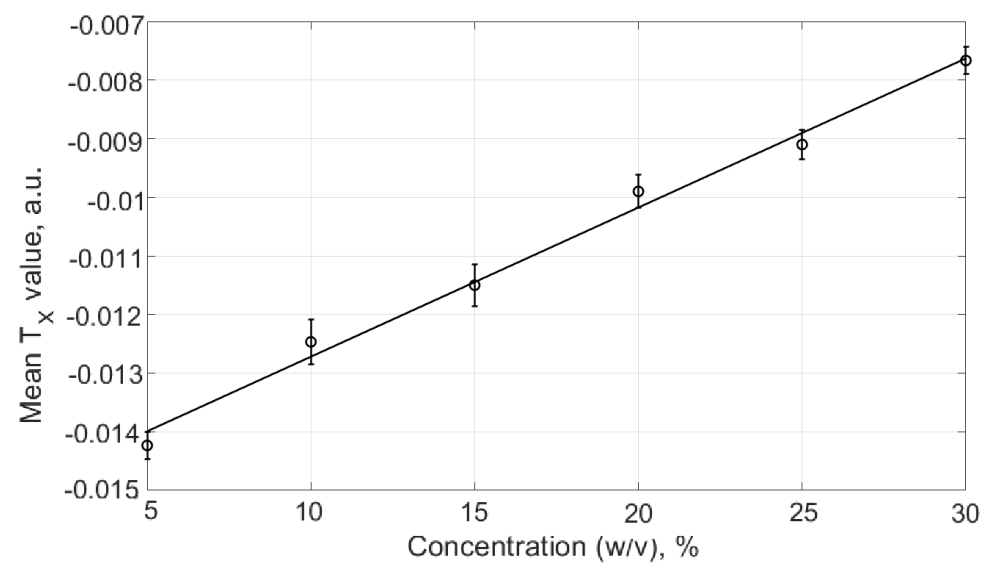

FIGURE 3 CW Tx values against the concentration of skimmed milk powder solutions using the CW NMR apparatus. The gradient of the fit is $2.5 E^{-4}$ a.u. $\cdot \%^{-1}$ mass

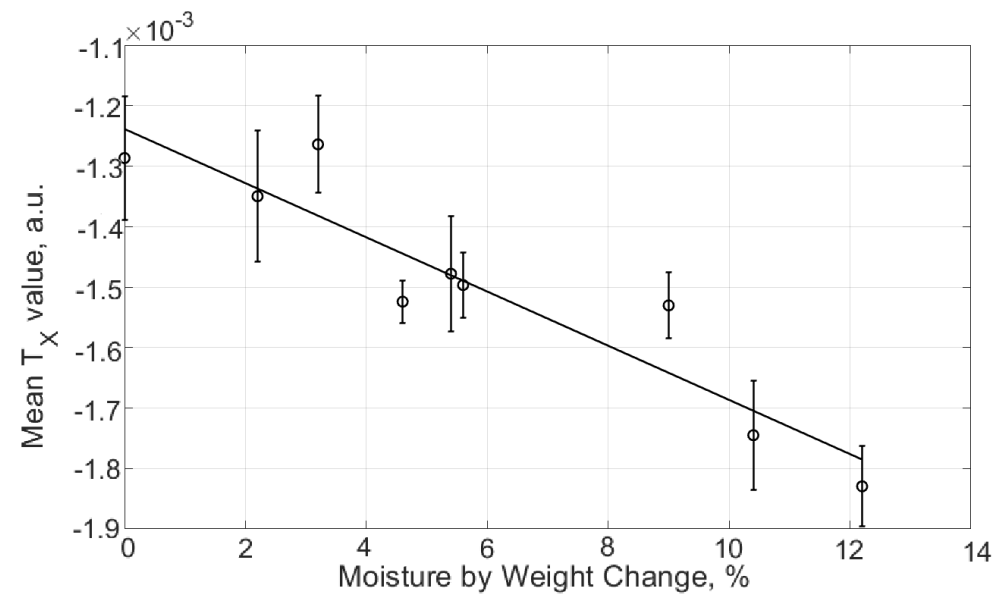

FIGURE 4 CW $\mathrm{T}_{\mathrm{x}}$ values against percentage moisture of milk powder made into $40 \% \mathrm{w} / \mathrm{v}$ solutions. The gradient of the fit is $-4.5 E^{-5}$ a.u. $\%^{-1}$ moisture 


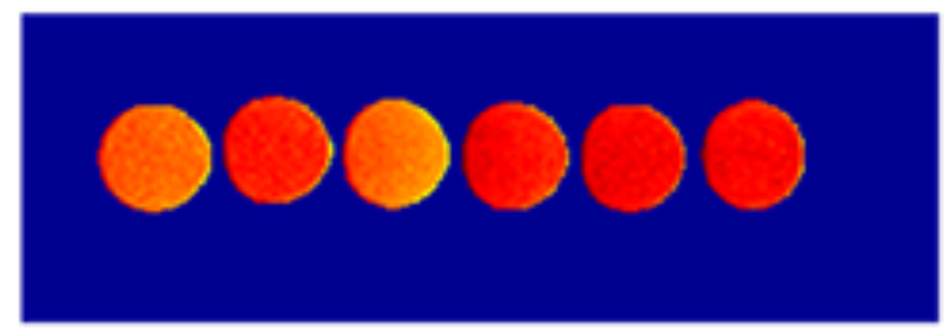

500

550 Time, ms 600

650

FIGURE 5 T1 map of milk powder solutions made from powders exposed to the humid environment chamber for (left to right) 0, 10, 20, 40,60 and 90 minutes. 